\title{
Evaluating the recent developments in palliative chemotherapy for metastatic colorectal cancer
}

\author{
Sun Kyung Baek ${ }^{1,{ }^{*}}$, Kyu Taek Lee ${ }^{2,}$, Sang Byung Bae ${ }^{2}$, and Sang-Cheol Lee ${ }^{2}$
}

\author{
${ }^{1}$ Department of Internal Medicine \\ Kyung Hee University Hospital, \\ Seoul; ${ }^{2}$ Department of Internal \\ Medicine, Soonchunhyang \\ University Cheonan Hospital, \\ Cheonan, Korea
}

Received: February 28, 2019

Accepted: May 21, 2019

\section{Correspondence to}

Sang-Cheol Lee, M.D.

Division of Hematology \&

Oncology, Department of Inter-

nal Medicine, Soonchunhyang

University Cheonan Hospital, 31

Suncheonhyang 6-gil, Dongnam-

gu, Cheonan 31151, Korea

Tel: +82-41-570-3803

Fax: +82-41-574-5762

E-mail: leptin72@gmail.com

https://orcid.org/0000-0003-0139-

9909

*'These authors contributed equally to this work.
The incidence of colorectal cancer (CRC) has increased. CRC is the third most common cancer and the fourth most common cause of cancer-related deaths in Korea. Palliative chemotherapy can be used to shrink tumors and ease symptoms caused by the cancer when cure is not possible. It is important to identify chemotherapeutic agents that can be used to effectively treat metastatic CRC (mCRC) and thus improve the survival and quality of life of patients with mCRC. This review aimed to evaluate the recent developments in palliative chemotherapy for $\mathrm{mCRC}$ and the biological or targeted agents used based on genetic alterations.

Keywords: Palliative; Chemothreapy; Metastatic colorectal cancer; Survival

\section{INTRODUCTION}

In Korea, colorectal cancer (CRC) is the third most common cancer and the fourth most common cause of cancer-related deaths [1]. Stage IV CRC accounted for $15 \%$ to $20 \%$ [2] and about $20 \%$ to $30 \%$ of cases of advanced relapsed CRC $[3,4]$. Effective therapeutic combinations can improve the survival and increase the rate of curative intent resections for metastatic CRC (mCRC). Patients with unresectable mCRC should be offered palliative chemotherapy. Cytotoxic chemotherapy of mCRC uses a combination of cytotoxic agents such as fluorouracil (FU), leucovorin (LV), capecitabine, irinote- can, and oxaliplatin for chemotherapy. Depending on whether cancer has RAS mutation (RAS-mt), biologic agents such as angiogenesis-targeting and epidermal growth factor receptor (EGFR)-targeting agents can be used as cytotoxic agents for combination chemotherapy. Recently, other molecular driver mutations such as BRAF (V6ooE) mutation (BRAF-mt), and human epidermal growth factor receptor 2 (HER2) amplification have led to the development of novel therapeutic targets. BRAF inhibitors, mitogen-activated protein kinase (MAPK) kinase (MEK) inhibitor, EGFR targeting drug, and HER2-targeting drug have been recently developed after recognizing molecular driver mutations. In 
2017, the U.S. Food and Drug Administration approved pembrolizumab and nivolumab for microsatellite instability-high (MSI-H) or mismatch repair-deficient CRC that has progressed following treatment with fluoropyrimidine, oxaliplatin, and irinotecan. This review aimed to evaluate the recent developments in palliative chemotherapy for mCRC and the agents used based on genetic alterations.

\section{CYTOTOXIC AGENTS}

In the past few years, effective treatment of advanced CRC only involved the use of FU. FU has a schedule-dependent mechanism of action [5]. FU could be delivered in various schedules via bolus injections or long-term infusions. When combined with LV, an anti-folate that can increase thymidylate synthetase inhibition, clinical outcomes can be improved [6]. De Gramont et al. [7] have developed a regimen that can be repeated every other week, employing a schedule of two consecutive daily 2-hour LV infusions followed by a FU bolus and 22-hour infusion for 2 consecutive days every 2 weeks (LV2FU2). The efficacy of this regimen in comparison with 5 days of 5 -FU/LV (FL) in improving response rate (RR) and progression-free survival (PFS) with decreased toxicities has been demonstrated [7]. In the 1990s, irinotecan and oxaliplatin were found to be effective against advanced CRC. Later, both regimens (folinic acid, FU, and oxaliplatin [FOLFOX] [8] and folinic acid, FU, and irinotecan [FOLFIRI]) [9] have demonstrated benefits as first-line therapy. Two sequences of these regimens such as FOLFOX followed by FOLFIRI and vice versa have shown similar efficacy [10]. Various 5 -FU prodrugs such as capecitabine [11,12], S-1 [13], and TAS-102 [14] have been developed with proven efficacy in place of 5 -FU alone or in combination for mCRC. Current first-line cytotoxic agents for mCRC include doublet combination of FOLFIRI [9], FOLFOX [8], and capecitabine with oxaliplatin (XELOX) [15] as well as triplet combination of FU, LV, oxaliplatin, and irinotecan (FOLFOXIRI) [16].

As initial therapy for mCRC, cytotoxic agents such as doublet combination of FOLFIRI, FOLFOX, and XELOX and triplet combination of FOLFOXIRI are recommended.

\section{COMBINATION WITH BIOLOGIC AGENTS}

\section{Angiogenesis-targeting agents}

Bevacizumab, a vascular endothelial growth factor A (VEGF-A) targeting monoclonal antibody, has been approved as first-line therapy for mCRC based on randomized phase III trial (AVF2107) in 2004. This trial compared irinotecan, $5 \mathrm{FU}$, and LV (IFL) with IFL plus bevacizumab, which showed superior RR, PFS, and overall survival (OS) [17]. Although a few trials failed to show the advantage of bevacizumab [18,19], some subsequent phase III trials demonstrated benefit of PFS or RR when bevacizumab was combined with first-line cytotoxic chemotherapy agents such as XELOX, FOLFOX, or capecitabine [20-22]. Bevacizumab also improved PFS and RR when combined with 5-FU triplet, 5-FOLFOXIRI compared with bevacizumab plus FOLFIRI [23].

The addition of bevacizumab, as biologic agent, to XELOX, FOLFOX, FOLFIRI, or FOLFOXIRI was the recommended first-line chemotherapy for mCRC. Anti-VEGF therapy as second-line chemotherapy included addition or continuation of bevacizumab or combination of another anti-angiogenetic agent with cytotoxic chemotherapy. Giantonio et al. [24] have demonstrated the advantage of OS, PFS, and RR from the addition of bevacizumab to FOLFOX in bevacizumab-naïve mCRC patients as second-line chemotherapy. The ML18147 study proved the survival benefit of continuing bevacizumab in patients who had progressed after first-line bevacizumab plus combination chemotherapy [25]. Aflibercept, a VEGF-a/b and placenta growth factor (PGF)-binding fusion protein, in combination with FOLFIRI in patients with $\mathrm{mCRC}$ who had progressed on first-line oxaliplatin-fluoropyrimidine therapy has resulted in modest improvement in PFS and OS compared with FOLFIRI plus placebo [26]. Ramucirumab, a VEGFR2 targeting monoclonal antibody, in combination with FOLFIRI for patients with $\mathrm{mCRC}$ who had progressed after first-line therapy with bevacizumab and oxaliplatin, has also resulted in improvements in PFS and OS [27].

If the patient's disease progresses after receiving firstline chemotherapy, bevacizumab plus alternative combination chemotherapy could be used as second-line therapy. In cases where patients progresses after receiving first-line oxaliplatin-based therapy, they could be considered for aflibercept or ramucirumab plus FOLFI- 
RI treatment.

Regorafenib, an oral multi-kinase inhibitor that blocks the activity of several protein kinases including VEGFR1/2/3, platelet-derived growth factor receptor (PDGFR), and fibroblast growth factor receptor (FGFR), has been proven effective against mCRC that had progressed after standard therapies by improving OS of patients [28].

Regorafenib monotherapy would be one of the options for mCRC patients after standard therapies.

\section{EGFR-targeting agents}

Several studies evaluating the effectiveness of EGFR-targeting drugs comprising cetuximab and panitumumab involved molecular studies to identify biomarkers. Although RAS-mt has been known as a biomarker of EGFR-targeting drugs, initial trials of cetuximab enrolled patients with immunohistochemically detectable EGFR expression. The NCI-CO17 study confirmed the efficacy of cetuximab as the best supportive care for chemotherapy-resistant mCRC and demonstrated that KRAS exon 2 mutation was a negative predictive biomarker [29,30]. Molecular analysis in subsequent clinical trials demonstrated that patients with KRAS exon 2 or other RAS-mt did not benefit from the addition of cetuximab or panitumumab [31-33]. RAS-mt occurs in exon 2 (codons 12 and 13), exon 3 (codons 59 and 61), and exon 4 (codons 117 and 146). Mutations in KRAS exon 2 codons account for about $80 \%$ of KRAS variants and about $42 \%$ of mCRC. Mutations in KRAS exons 3 and 4 and NRAS exons 2, 3 , and 4 account for around 10\% of mCRC cases [34].

Two early clinical trials at first-line setting of mCRC doubted the efficacy of cetuximab in combination with oxaliplatin-based chemotherapy [35,36]. The COIN study used FOLFOX or XELOX according to the physician's choice. Adding cetuximab to these regimens remained unbeneficial [35]. The addition of cetuximab to XELOX resulted in the reduction of dose intensity and increase in capecitabine toxicities. However, FOLFOX with cetuximab improved PFS compared with FOLFOX in subgroup analysis. The CRISTAL study investigated the addition of cetuximab to FOLFIRI. Addition of cetuximab improved the RR, PFS, and OS [37,38]. Panitumumab, a fully human immunoglobulin G2 antibody, in combination with FOFLOX chemotherapy in KRAS wild-type patients showed efficacy in improving PFS
[39].

Cetuximab and panitumumab with FOLFOX or FOLFIRI can be used as first-line chemotherapy in mCRC patients with RAS wild type. In addition, cetuximab could be considered in combination with irinotecan in patients with irinotecan-refractory CRC.

\section{Selection of biologic agent in RAS wild type}

Which among the biologic agents (bevacizumab and cetuximab or panitumumab) are more effective against RAS wild type? Three studies have been conducted to address this question. However, the superiority of either bevacizumab or cetuximab/panitumumab has not been established yet. A randomized phase III study, FIRE-3 ( $\mathrm{n}=592)$, compared cetuximab and bevacizumab in combination with FOLFIRI chemotherapy in patients with KRAS wild type. It found no significant difference in RR as primary endpoint [40]. A randomized phase II study, the PEAK $(n=285)$, compared the effectiveness of panitumumab and bevacizumab in combination with FOLFOX in MCRC patients with KRAS wild type. It found no significant difference in PFS as primary endpoint [41]. A randomized phase III study, CALGB/SWOG 80405 trial $(\mathrm{n}=1,137)$, also involved mCRC patients with KRAS wild type. Results showed that there was no significant difference in OS as primary endpoint between patients treated with cetuximab and those treated with bevacizumab combined with mFOLFOX6 or FOLFIRI as first-line therapy [42]. Although the FIRE-3 study and PEAK study reported OS benefit in cetuximab or panitumumab group, no significant difference in OS, PFS, or RR was observed among these groups in the CALGB/SWOG 80405 trial, the largest among the three trials, which enrolled 1,000 patients.

Either anti-angiogenic agent such as bevacizumab or anti-EGFR inhibitors including cetuximab or panitumumab with FOLFOX/XELOX or FOLFIRI would be recommended as the first-line chemotherapy in mCRC patients with RAS wild type.

In addition, the CALGB/SWOG 80405 trial suggested that the primary tumor location might be a predictive biomarker as well as a prognostic biomarker in patients with KRAS wild type. A meta-analysis of first-line therapy for RAS wild type demonstrated that patients with left side colon cancer had a significant survival benefit 
from anti-EGFR agents than with anti-VEGF agent added to standard chemotherapy $[43,44]$.

In left side colon cancer with RAS wild type, anti-EGFR inhibitors are preferred as biologic agents combined with FOLFOX/XELOX or FOLFIRI as first-line chemotherapy.

\section{THERAPEUTIC GOALS}

\section{Potentially resectable liver metastatic disease}

The 5-year survival rate of patients with mCRC and liver metastasis that underwent metastasectomy and primary lesion resection was $35 \%$ to $60 \%$ [45]. A previous prospective randomized trial proved the benefit of adjuvant FL chemotherapy following Ro hepatic metastasectomy [46]. A pooled analysis showed the same result [47]. The EORTC 40983 study compared perioperative chemotherapy with FOLFOX 4 and surgery and surgery alone for resectable liver metastases from mCRC [48]. This study proved that FOLFOX as perioperative chemotherapy effectively reduced the risk of adverse events that could affect PFS. However, the addition of anti-EGFR antibody to FOLFOX in these patients did not improve the outcomes [48]. Whether surgery should be performed first or the exact number of cycles of preoperative chemotherapy remains unclear. Prolonged preoperative chemotherapy could increase the risk of postoperative complications after major liver resection as it is associated with liver problems such as fatty liver, steatohepatitis, or sinusoidal injury due to cytotoxic chemotherapy [49]. Karoui et al. [50] reported that morbidity in the chemotherapy group increased among patients receiving six or more cycles of chemotherapy.

Primary lesion resection and metastasectomy with perioperative chemotherapy should be considered in mCRC patients with potentially resectable liver metastasis. However, the effect of using a combination of biologic agents remains unclear.

\section{Unresectable disease}

Some prospective studies have reported the downsizing of patients with colorectal liver metastases for rescue surgery following combination chemotherapy, with Ro resection of $20 \%$ to $50 \%$ and median OS of 17 to 48.8 months [51]. Conversion chemotherapy administered in patients with initially unresectable liver metastasis increased the resectability of liver metastasis and improved their survival [52]. The combination of either oxaliplatin- or irinotecan-based chemotherapy and EGFR antibody for RAS-wild type or anti-VEGF antibody can improve Ro resection rates [53,54]. FOLFOXIRI with bevacizumab can improve tumor RR, resection rate, and PFS than FOLFOX with bevacizumab [51].

Effective conversion chemotherapy and resection of primary lesion and liver metastasis could improve the outcome of mCRC patients with unresectable liver metastasis. The use of a combination of cytotoxic chemotherapy and biologic agents as doublet or bevacizumab plus FU, LV, and oxaliplatin are recommended to convert the unresectable tumor to resectable tumor.

\section{Optimization of chemotherapy}

\section{Subsequence therapy}

For mCRC patients with RAS wild type who were treated with anti-angiogenic agent such as bevacizumab or anti-EGFR inhibitor including cetuximab or panitumumab plus FOLFOX/XELOX or FOLFIRI or bevacizumab plus FOLFOXIRI as first-line chemotherapy and vice versa, cytotoxic agents could be considered. Cytotoxic agents with two sequences of FOLFOX/XELOX followed FOLFIRI and vice versa showed similar efficacy [10]. If FOLFOX/XELOX plus bevacizumab was used as the first-line chemotherapy, FOLFIRI plus bevacizumab, ramucirumab, or aflibercept could be considered as the second-line chemotherapy. If FOLFOX/ XELOX plus cetuximab or panitumumab was used as the first-line chemotherapy, FOLFIRI plus bevacizum$\mathrm{ab}$ or aflibercep could be considered as the second-line chemotherapy. If FOLFIRI plus bevacizumab or plus cetuximab or panitumumab was used as the first-line chemotherapy, FOLFOX plus bevacizumab could be considered as the second-line chemotherapy. If patients who were resistant to irinotecan did not receive cetuximab previously, they could be considered for cetuximab plus irinotecan therapy.

In mCRC patients with RAS mutant type, bevacizumab with FOLFOX/XELOX, FOLFIRI, or FOLFOXIRI could be used because of no efficacy of anti EGFR inhibitors. If FOLFOX/XELOX plus bevacizumab was used as the first-chemotherapy, FOLFIRI plus bevacizumab, ramucirumab, or aflibercept could be considered as the sec- 
ond-line chemotherapy. If FOLFIRI plus bevacizumab was used as the first-line chemotherapy, FOLFOX plus bevacizumab could be considered as the second-line chemotherapy.

Regardless of RAS-mt status, regorafenib or TAS 102 could be considered in patients with MCRC who are resistant to oxaliplatin and irinotecan.

\section{Continuous or intermittent therapy}

The optimal duration of palliative chemotherapy for unresectable disease that does not progress remains controversial. Oxaliplatin causes cumulative neurotoxicity, which generally induces discontinuation of chemotherapy. OTIMOXı study has investigated whether continuous therapy provides better outcomes than intermittent therapy to achieve better response after chemotherapy. In this study, 620 previously untreated patients were randomly assigned to FOLFOX 4 every 2 weeks until progression (arm A), or $\mathrm{FOLFOX}_{7}$ for 6 cycles only, followed by reinduction of oxaliplatin at the time of progression after 12 cycles of non-oxaliplatin-containing regimen (arm B). Median survival was similar between the two groups. However, patients in arm B had lower risk of grade 3/4 toxicity during cycles 6 to 18 [55].

Considering the increased toxicities due to continuous treatment, intermittent therapy could be used as an alternative treatment.

\section{Maintenance therapy}

The efficacy of bevacizumab alone or bevacizumab with FL or capecitabine combined with FOLFOX/XELOX was investigated. CAIRO 3 study was conducted to determine the efficacy of capecitabine plus bevacizumab in patients with stable disease or evaluate the results after six cycles of XELOX plus bevacizumab. The maintenance therapy group showed longer PFS [56]. Comparing treatment break and continuation of bevacizumab alone, the continuous administration of fluoropyrimidine and bevacizumab until progression after first-line combination therapy improved patients' PFS [57].

Administering maintenance therapy with 5-FU and bevacizumab after first-line FOLFOX/XELOX with bevacizumab was found to be effective.

\section{SPECIAL CONSIDERATIONS}

\section{Other chromosomal instability}

BRAF-mt and HER2 amplifications account for about $5 \%$ and $3 \%$ of $\mathrm{mCRC}$, respectively. Because RAS-mt, BRAF-mt, and HER2 amplifications are mutually exclusive [35,58], in patients with RAS wild-type disease, the rate of BRAF-mt or HER2 amplification increased. Use of BRAF inhibitors and HER2-targeting drugs improved the outcomes of patients with mCRC.

BRAF-mt is more prevalent in patients with proximal colon tumors. It has unique pathologic features such as poorer differentiation, mucinous histology, and MSI [59]. BRAF-mt confers worse prognosis in metastatic setting. However, it does not predict response to standard chemotherapy. The predictive value of anti-EGFR antibodies for patients with BRAF-mt remains controversial. However, results suggested that patients with BRAF-mt might not benefit from cetuximab or panitumumab. The European Society for Medical Oncology (ESMO) guideline recommend triplet chemotherapy combined with bevacizumab to manage aggressive BRAF-mt mCRC in fit patients [6o].

BRAF, a modulator of the MAPK pathway, has recently emerged as a promising new target for the treatment of CRC. Monotherapy using BRAF inhibitors has a poor RR (5\%) [61]. This primary resistance results from feedback signals that can reactivate MAPK signaling. To overcome this resistance, BRAF inhibitors have been studied in combination with inhibitors of MARK pathway mediators. Dual inhibition of BRAF and MEK such as in BRAF-mt melanoma, dual inhibition of EGFR and BRAF with or without irinotecan, or use of triple combination therapy has been studied with promising data [59].

Although the incidence of HER2 amplification of mCRC is known to be below $5 \%$, in patients with both RAS and BRAF wild-type diseases, a HER2 amplification rate of $14 \%$ has been reported [62]. Amplification of the HER gene or overexpression of its protein product has been successfully treated in patients with other types of cancer, most notably breast cancer and gastric adenocarcinomas. HER2 target agents including trastuzumab, lapatinib, and pertuzumab have become important treatment options for these cancers. The HERACLES study demonstrated the efficacy of a com- 
bination of trastuzumab and lapatinib in patients with HER2-positive mCRC who were resistance to standard therapy [63].

\section{Microsatellite instability}

MSI is the molecular fingerprint of a deficient mismatch repair system. Approximately $15 \%$ of CRC cases display MSI owing either to epigenetic silencing of mutL homolog 1 (MLH1) or germline mutation in one of mismatch-repair (MMR) genes: $\mathrm{MLH1}, \mathrm{MSH} 2$, MSH6, or PMS2. MSI is diagnosed on the basis of the variable length of DNA microsatellites. It allows mutations to be accumulated at many times and facilitates MSI neoplastic progression. PD-1 inhibitor in MSI-H or mismatch repair-deficient mCRC has demonstrated clinical benefit [64]. Nivolumab has demonstrated efficacy in CRC that has progressed following treatment with a fluoropyrimidine, oxaliplatin, and irinotecan, showing clinical benefit [65].

Most patients with mCRC are mismatch repair proficient and microsatellite stable. Immune checkpoint inhibitors have yet to show clinical activity in these patients. Immune checkpoint inhibitors combined with synergistic drugs such as MEK inhibitors have been tried in these patients to improve tumor immune recognition or promote immune cell accumulation [66].

\section{CONCLUSIONS}

New development of chemotherapeutic agent and targeted agents including anti-angiogenic agents or anti EGFR inhibitors and appropriate surgical treatment have improved the prognosis of mCRC. Recently, research on target agents such as BRAF, HER2, MSI-H, and new targeted agents for other abnormalities have been investigated with proven efficacy.

\section{Conflict of interest}

No potential conflict of interest relevant to this article was reported.

\section{Acknowledgments}

This research was supported by Soonchunhyang University Research Fund.

\section{REFERENCES}

1. Jung KW, Won YJ, Kong HJ, et al. Cancer statistics in Korea: incidence, mortality, survival, and prevalence in 2012. Cancer Res Treat 2015;47:127-141.

2. Watanabe T, Itabashi M, Shimada Y, et al. Japanese Society for Cancer of the Colon and Rectum (JSCCR) guidelines 2010 for the treatment of colorectal cancer. Int J Clin Oncol 2012;17:1-29.

3. Andre T, Boni C, Mounedji-Boudiaf L, et al. Oxaliplatin, fluorouracil, and leucovorin as adjuvant treatment for colon cancer. N Engl J Med 2004;350:2343-2351.

4. Seo SI, Lim SB, Yoon YS, et al. Comparison of recurrence patterns between $\leq 5$ years and $>5$ years after curative operations in colorectal cancer patients. J Surg Oncol 2013;108:9-13.

5. Sobrero AF, Aschele C, Bertino JR. Fluorouracil in colorectal cancer: a tale of two drugs: implications for biochemical modulation. J Clin Oncol 1997;15:368-381.

6. Modulation of fluorouracil by leucovorin in patients with advanced colorectal cancer: evidence in terms of response rate. Advanced Colorectal Cancer Meta-Analysis Project. J Clin Oncol 1992;10:896-903.

7. de Gramont A, Bosset JF, Milan C, et al. Randomized trial comparing monthly low-dose leucovorin and fluorouracil bolus with bimonthly high-dose leucovorin and fluorouracil bolus plus continuous infusion for advanced colorectal cancer: a French intergroup study. J Clin Oncol 1997;15:808-815.

8. de Gramont A, Figer A, Seymour M, et al. Leucovorin and fluorouracil with or without oxaliplatin as first-line treatment in advanced colorectal cancer. J Clin Oncol 2000;18:2938-2947.

9. Douillard JY, Cunningham D, Roth AD, et al. Irinotecan combined with fluorouracil compared with fluorouracil alone as first-line treatment for metastatic colorectal cancer: a multicentre randomised trial. Lancet 2000;355:10411047 .

10. Tournigand C, Andre T, Achille E, et al. FOLFIRI followed by FOLFOX6 or the reverse sequence in advanced colorectal cancer: a randomized GERCOR study. J Clin Oncol 2004;22:229-237.

11. Hoff PM, Ansari R, Batist G, et al. Comparison of oral capecitabine versus intravenous fluorouracil plus leucovorin as first-line treatment in 605 patients with metastatic colorectal cancer: results of a randomized phase III study. 
J Clin Oncol 2001;19:2282-2292.

12. Van Cutsem E, Twelves C, Cassidy J, et al. Oral capecitabine compared with intravenous fluorouracil plus leucovorin in patients with metastatic colorectal cancer: results of a large phase III study. J Clin Oncol 2001;19:40974106.

13. Kwakman JJM, Simkens LHJ, van Rooijen JM, et al. Randomized phase III trial of S-1 versus capecitabine in the first-line treatment of metastatic colorectal cancer: SALTO study by the Dutch Colorectal Cancer Group. Ann Oncol 2017;28:1288-1293.

14. Mayer RJ, Van Cutsem E, Falcone A, et al. Randomized trial of TAS-102 for refractory metastatic colorectal cancer. N Engl J Med 2015;372:1909-1919.

15. Cassidy J, Clarke S, Diaz-Rubio E, et al. Randomized phase III study of capecitabine plus oxaliplatin compared with fluorouracil/folinic acid plus oxaliplatin as firstline therapy for metastatic colorectal cancer. J Clin Oncol 2008;26:2006-2012.

16. Falcone A, Ricci S, Brunetti I, et al. Phase III trial of infusional fluorouracil, leucovorin, oxaliplatin, and irinotecan (FOLFOXIRI) compared with infusional fluorouracil, leucovorin, and irinotecan (FOLFIRI) as first-line treatment for metastatic colorectal cancer: the Gruppo Oncologico Nord Ovest. J Clin Oncol 2007;25:1670-1676.

17. Hurwitz H, Fehrenbacher L, Novotny W, et al. Bevacizumab plus irinotecan, fluorouracil, and leucovorin for metastatic colorectal cancer. N Engl J Med 2004;350:2335-2342.

18. Passardi A, Nanni O, Tassinari D, et al. Effectiveness of bevacizumab added to standard chemotherapy in metastatic colorectal cancer: final results for first-line treatment from the ITACa randomized clinical trial. Ann Oncol 2015;26:1201-1207.

19. Stathopoulos GP, Batziou C, Trafalis D, et al. Treatment of colorectal cancer with and without bevacizumab: a phase III study. Oncology 2010;78:376-381.

20. Cunningham D, Lang I, Marcuello E, et al. Bevacizumab plus capecitabine versus capecitabine alone in elderly patients with previously untreated metastatic colorectal cancer (AVEX): an open-label, randomised phase 3 trial. Lancet Oncol 2013;14:1077-1085.

21. Saltz LB, Clarke S, Diaz-Rubio E, et al. Bevacizumab in combination with oxaliplatin-based chemotherapy as first-line therapy in metastatic colorectal cancer: a randomized phase III study. J Clin Oncol 2008;26:2013-2019. 22. Tebbutt NC, Wilson K, Gebski VJ, et al. Capecitabine, bevacizumab, and mitomycin in first-line treatment of metastatic colorectal cancer: results of the Australasian Gastrointestinal Trials Group Randomized Phase III MAX Study. J Clin Oncol 2010;28:3191-3198.

23. Loupakis F, Cremolini C, Masi G, et al. Initial therapy with FOLFOXIRI and bevacizumab for metastatic colorectal cancer. N Engl J Med 2014;371:1609-1618.

24. Giantonio BJ, Catalano PJ, Meropol NJ, et al. Bevacizum$\mathrm{ab}$ in combination with oxaliplatin, fluorouracil, and leucovorin (FOLFOX 4 ) for previously treated metastatic colorectal cancer: results from the Eastern Cooperative Oncology Group Study E3200. J Clin Oncol 2007;25:15391544 .

25. Bennouna J, Sastre J, Arnold D, et al. Continuation of bevacizumab after first progression in metastatic colorectal cancer (ML18147): a randomised phase 3 trial. Lancet Oncol 2013;14:29-37.

26. Van Cutsem E, Tabernero J, Lakomy R, et al. Addition of aflibercept to fluorouracil, leucovorin, and irinotecan improves survival in a phase III randomized trial in patients with metastatic colorectal cancer previously treated with an oxaliplatin-based regimen. J Clin Oncol 2012;30:34993506.

27. Tabernero J, Yoshino T, Cohn AL, et al. Ramucirumab versus placebo in combination with second-line FOLFI$\mathrm{RI}$ in patients with metastatic colorectal carcinoma that progressed during or after first-line therapy with bevacizumab, oxaliplatin, and a fluoropyrimidine (RAISE): a randomised, double-blind, multicentre, phase 3 study. Lancet Oncol 2015;16:499-508.

28. Grothey A, Van Cutsem E, Sobrero A, et al. Regorafenib monotherapy for previously treated metastatic colorectal cancer (CORRECT): an international, multicentre, randomised, placebo-controlled, phase 3 trial. Lancet 2013;381:303-312.

29. Jonker DJ, O'Callaghan CJ, Karapetis CS, et al. Cetuximab for the treatment of colorectal cancer. N Engl J Med 2007;357:2040-2048.

30. Karapetis CS, Khambata-Ford S, Jonker DJ, et al. K-ras mutations and benefit from cetuximab in advanced colorectal cancer. N Engl J Med 2008;359:1757-1765.

31. Bokemeyer C, Kohne CH, Ciardiello F, et al. FOLFOX 4 plus cetuximab treatment and RAS mutations in colorectal cancer. Eur J Cancer 2015;51:1243-1252.

32. Douillard JY, Oliner KS, Siena S, et al. Panitumumab-FOLFOX 4 treatment and RAS mutations in colorectal 
cancer. N Engl J Med 2013;369:1023-1034.

33. Van Cutsem E, Lenz HJ, Kohne CH, et al. Fluorouracil, leucovorin, and irinotecan plus cetuximab treatment and RAS mutations in colorectal cancer. J Clin Oncol 2015;33:692-700.

34. Misale S, Di Nicolantonio F, Sartore-Bianchi A, Siena S, Bardelli A. Resistance to anti-EGFR therapy in colorectal cancer: from heterogeneity to convergent evolution. Cancer Discov 2014;4:1269-1280.

35. Maughan TS, Adams RA, Smith CG, et al. Addition of cetuximab to oxaliplatin-based first-line combination chemotherapy for treatment of advanced colorectal cancer: results of the randomised phase 3 MRC COIN trial. Lancet 2011;377:2103-2114.

36. Tveit KM, Guren T, Glimelius B, et al. Phase III trial of cetuximab with continuous or intermittent fluorouracil, leucovorin, and oxaliplatin (Nordic FLOX) versus FLOX alone in first-line treatment of metastatic colorectal cancer: the NORDIC-VII study. J Clin Oncol 2012;30:17551762.

37. Van Cutsem E, Kohne CH, Hitre E, et al. Cetuximab and chemotherapy as initial treatment for metastatic colorectal cancer. N Engl J Med 2009;360:1408-1417.

38. Van Cutsem E, Kohne CH, Lang I, et al. Cetuximab plus irinotecan, fluorouracil, and leucovorin as first-line treatment for metastatic colorectal cancer: updated analysis of overall survival according to tumor KRAS and BRAF mutation status. J Clin Oncol 2011;29:2011-2019.

39. Douillard JY, Siena S, Cassidy J, et al. Randomized, phase III trial of panitumumab with infusional fluorouracil, leucovorin, and oxaliplatin (FOLFOX4) versus FOLFOX 4 alone as first-line treatment in patients with previously untreated metastatic colorectal cancer: the PRIME study. J Clin Oncol 2010;28:4697-4705.

40. Stintzing S, Modest DP, Rossius L, et al. FOLFIRI plus cetuximab versus FOLFIRI plus bevacizumab for metastatic colorectal cancer (FIRE-3): a post-hoc analysis of tumour dynamics in the final RAS wild-type subgroup of this randomised open-label phase 3 trial. Lancet Oncol 2016;17:1426-1434.

41. Schwartzberg LS, Rivera F, Karthaus M, et al. PEAK: a randomized, multicenter phase II study of panitumumab plus modified fluorouracil, leucovorin, and oxaliplatin (mFOLFOX6) or bevacizumab plus mFOLFOX6 in patients with previously untreated, unresectable, wild-type KRAS exon 2 metastatic colorectal cancer. J Clin Oncol
2014;32:2240-2247

42. Venook AP, Niedzwiecki D, Lenz HJ, et al. Effect of first-line chemotherapy combined with cetuximab or bevacizumab on overall survival in patients with KRAS wild-type advanced or metastatic colorectal cancer: a randomized clinical trial. JAMA 2017;317:2392-2401.

43. Arnold D, Lueza B, Douillard JY, et al. Prognostic and predictive value of primary tumour side in patients with RAS wild-type metastatic colorectal cancer treated with chemotherapy and EGFR directed antibodies in six randomized trials. Ann Oncol 2017;28:1713-1729.

44. Tejpar S, Stintzing S, Ciardiello F, et al. Prognostic and predictive relevance of primary tumor location in patients with RAS wild-type metastatic colorectal cancer: retrospective analyses of the CRYSTAL and FIRE-3 trials. JAMA Oncol 2017;3:194-201.

45. Morgan CT, Cho CS. Systemic chemotherapy for resectable hepatic colorectal metastases: adjuvant, neoadjuvant, or not at all? Curr Surg Rep 2014;2:52.

46. Portier G, Elias D, Bouche O, et al. Multicenter randomized trial of adjuvant fluorouracil and folinic acid compared with surgery alone after resection of colorectal liver metastases: FFCD ACHBTH AURC 9002 trial. J Clin Oncol 2006;24:4976-4982.

47. Mitry E, Fields AL, Bleiberg H, et al. Adjuvant chemotherapy after potentially curative resection of metastases from colorectal cancer: a pooled analysis of two randomized trials. J Clin Oncol 2008;26:4906-4911.

48. Primrose J, Falk S, Finch-Jones M, et al. Systemic chemotherapy with or without cetuximab in patients with resectable colorectal liver metastasis: the New EPOC randomised controlled trial. Lancet Oncol 2014;15:601-611.

49. Chun YS, Laurent A, Maru D, Vauthey JN. Management of chemotherapy-associated hepatotoxicity in colorectal liver metastases. Lancet Oncol 2009;10:278-286.

50. Karoui M, Penna C, Amin-Hashem M, et al. Influence of preoperative chemotherapy on the risk of major hepatectomy for colorectal liver metastases. Ann Surg 2006;243:1-7.

51. Gruenberger T, Bridgewater J, Chau I, et al. Bevacizumab plus mFOLFOX-6 or FOLFOXIRI in patients with initially unresectable liver metastases from colorectal cancer: the OLIVIA multinational randomised phase II trial. Ann Oncol 2015;26:702-708.

52. Blazer DG 3rd, Kishi Y, Maru DM, et al. Pathologic response to preoperative chemotherapy: a new outcome end point after resection of hepatic colorectal metastases. 
J Clin Oncol 2008;26:5344-5351.

53. Folprecht G, Gruenberger T, Bechstein W, et al. Survival of patients with initially unresectable colorectal liver metastases treated with FOLFOX/cetuximab or FOLFIRI/ cetuximab in a multidisciplinary concept (CELIM study). Ann Oncol 2014;25:1018-1025.

54. Ye LC, Liu TS, Ren L, et al. Randomized controlled trial of cetuximab plus chemotherapy for patients with KRAS wild-type unresectable colorectal liver-limited metastases. J Clin Oncol 2013;31:1931-1938.

55. Tournigand C, Cervantes A, Figer A, et al. OPTIMOXı: a randomized study of FOLFOX 4 or FOLFOX 7 with oxaliplatin in a stop-and-go fashion in advanced colorectal cancer: a GERCOR study. J Clin Oncol 2006;24:394-400.

56. Simkens LH, van Tinteren H, May A, et al. Maintenance treatment with capecitabine and bevacizumab in metastatic colorectal cancer (CAIRO3): a phase 3 randomised controlled trial of the Dutch Colorectal Cancer Group. Lancet 2015;385:1843-1852.

57. Hegewisch-Becker S, Graeven U, Lerchenmuller CA, et al. Maintenance strategies after first-line oxaliplatin plus fluoropyrimidine plus bevacizumab for patients with metastatic colorectal cancer (AIO 0207): a randomised, non-inferiority, open-label, phase 3 trial. Lancet Oncol 2015;16:1355-1369.

58. Cancer Genome Atlas Network. Comprehensive molecular characterization of human colon and rectal cancer. Nature 2012;487:330-337.
59. Sanz-Garcia E, Argiles G, Elez E, Tabernero J. BRAF mutant colorectal cancer: prognosis, treatment, and new perspectives. Ann Oncol 2017;28:2648-2657.

6o. Van Cutsem E, Cervantes A, Adam R, et al. ESMO consensus guidelines for the management of patients with metastatic colorectal cancer. Ann Oncol 2016;27:1386-1422.

61. Kopetz S, Desai J, Chan E, et al. Phase II pilot study of vemurafenib in patients with metastatic BRAF-mutated colorectal cancer. J Clin Oncol 2015;33:4032-4038.

62. Greally M, Kelly CM, Cercek A. HER2: an emerging target in colorectal cancer. Curr Probl Cancer 2018;42:560-571.

63. Sartore-Bianchi A, Trusolino L, Martino C, et al. Dual-targeted therapy with trastuzumab and lapatinib in treatment-refractory, KRAS codon 12/13 wild-type, HER2-positive metastatic colorectal cancer (HERACLES): a proofof-concept, multicentre, open-label, phase 2 trial. Lancet Oncol 2016;17:738-746.

64. Le DT, Uram JN, Wang H, et al. PD-1 blockade in tumors with mismatch-repair deficiency. N Engl J Med 2015;372:2509-2520.

65. Overman MJ, Lonardi S, Wong KYM, et al. Durable clinical benefit with nivolumab plus ipilimumab in DNA mismatch repair-deficient/microsatellite instability-high metastatic colorectal cancer. J Clin Oncol 2018;36:773-779.

66. Lee JJ, Chu E. Recent advances in the clinical development of immune checkpoint blockade therapy for mismatch repair proficient (pMMR)/non-MSI-H metastatic colorectal cancer. Clin Colorectal Cancer 2018;17:258-273. 